\title{
Transtornos mentais comuns em estudantes universitários de um Centro de
}

\section{Instrução Militar}

\author{
Common mental disorders in college students of a Military Education Center \\ Trastornos mentales comunes em estudiantes universitarios em un Centro de Instrucción Militar
}

Recebido: 30/05/2021 | Revisado: 09/06/2021 | Aceito: 13/06/2021 | Publicado: 26/06/2021

\author{
Aline Nogueira Santos \\ ORCID: https://orcid.org/0000-0003-4455-266X \\ Marinha do Brasil, Brasil \\ E-mail: alinenogueira@id.uff.br \\ Desireé Maia Neves \\ ORCID: https://orcid.org/0000-0003-3732-639X \\ Marinha do Brasil, Brasil \\ E-mail: maia.desiree@gmail.com \\ Yan de Jesus Ferreira Lima \\ ORCID: https://orcid.org/0000-0002-0247-7297 \\ Marinha do Brasil, Brasil \\ E-mail: yandejesus1@hotmail.com \\ Beatriz Tosé Agathão \\ ORCID: https://orcid.org/0000-0003-3288-5504 \\ Marinha do Brasil, Brasil \\ E-mail: beatrizagathao1705@gmail.com
}

\begin{abstract}
Resumo
Este artigo visa avaliar a frequência de transtorno mental comum (TMC) entre os alunos de graduação de uma escola de formação militar, de acordo com características sociodemográficas e aspectos comportamentais. O estudo foi realizado em 2020, com 194 alunos entre 17 e 25 anos na Escola de Formação de Oficiais da Marinha Mercante (EFOMM) localizada no Rio de Janeiro. Para avaliar a presença de TMC foi usada a versão reduzida do General Health Questionnaire (GHQ-12). A análise dos dados apontou que os TMC estão presentes em $71.1 \%$ dos alunos participantes, sendo a maior frequência entre as jovens do sexo feminino, estudantes do segundo ano, mais velhos, entre os alunos não brancos (pardos, pretos e amarelos), baixa posição socioeconômica, não heterossexuais e os que sofrem maior influência da fé. Os resultados deste estudo podem auxiliar a identificar subgrupos mais vulneráveis, além da criação de medidas de prevenção e gestão mais focalizadas no ambiente universitário.
\end{abstract}

Palavras-chave: Transtornos mentais; Estudantes; Epidemiologia; Saúde mental.

\begin{abstract}
This article aims to assess the frequency of common mental disorder (CMD) among undergraduate students at a military training school, according to sociodemographic characteristics and behavioral aspects. The study was carried out in 2020, with 194 students between 17 and 25 years of age at a Merchant Marine officers training school (EFOMM). The reduced version of the General Health Questionnaire (GHQ-12) was used to assess the presence of CMD. Data analysis showed that CMDs are present in $71.1 \%$ of participating students, with a higher frequency among young women, second year students, older, non-white students (brown, black and yellow), low socioeconomic status, non-heterosexuals and those who are most influenced by faith. The results of this study can help to identify more vulnerable subgroups, in addition to creating more focused prevention and management measures in the university environment.
\end{abstract}

Keywords: Mental disorders; Students; Epidemiology; Mental health.

\section{Resumen}

Este artículo tiene como objetivo evaluar la frecuencia de los trastornos mentales comunes (TMC) entre los estudiantes universitarios en una escuela de entrenamiento militar, según las características sociodemográficas y los aspectos de comportamiento. El estudio se llevó a cabo en 2020, con 194 estudiantes entre 17 y 25 años en una escuela de formación de oficiales de la Marina Mercante (EFOMM). La versión reducida del General Health Questionnaire (GHQ-12) se utilizó para evaluar la presencia de TMC. El análisis de datos mostró que los TMC están presentes en el 71.1\% de los estudiantes participantes, con una mayor frecuencia entre las mujeres jóvenes, estudiantes de segundo año, mayores, entre los estudiantes no blancos (pardo, negro y amarillo), bajo nivel socioeconómico, no heterosexuales y aquellos que están más influenciados por la fe. Los resultados de este estudio pueden ayudar a identificar subgrupos más vulnerables, además de crear medidas de prevención y manejo más enfocadas en el ámbito universitario.

Palabras clave: Trastornos mentales; Estudiantes; Epidemiología; Salud mental. 


\section{Introdução}

Os transtornos mentais não psicóticos possuem alta prevalência na população geral, com frequências entre $25.9 \%$ e $32.6 \%$ (Steel, 2014), e são conhecidos, portanto, como transtornos mentais comuns (TMC). Caracterizam-se pela presença de dois principais sintomas subjacentes: sintomas depressivos e ansiosos (Goldberg, 1994; Lopes et al., 2016; Agathão, Lopes, Cunha \& Sichieri, 2020). Os sintomas dos TMCs apresentam impacto no humor ou sentimentos das pessoas afetadas e variam em termos de gravidade (de leve a grave) e duração (de meses a anos). Segundo a publicação Depression and Other Common Mental Disorders: Global Health Estimates (World Health Organization [WHO], 2017), estima-se que 4.4\% da população global sofra de transtornos depressivos e 3.6\% de transtornos de ansiedade. No Brasil, essa porcentagem chega a 5.8\% e 9.3\% da população, respectivamente.

De acordo com a análise de Marinho et al. (2018), com base nos dados do Global Burden Disease Study (GBD) (2016), transtornos depressivos e ansiosos correspondem respectivamente à quinta e sexta causas de anos vividos com incapacidade. Em seu estado mais severo, a depressão pode levar ao suicídio' sendo esta a segunda principal causa de óbito em jovens de 15 a 29 anos (WHO, 2017).

Em universitários, a depressão está ligada ao baixo desempenho acadêmico, instabilidade em relacionamentos, tentativas e pensamentos suicidas e baixo desempenho no trabalho entre aqueles com jornada dupla (que estudam e trabalham) (Ibrahim, Kelly, Adams \& Glazebrook, 2013). Vale ressaltar que a ocorrência de transtornos mentais em jovens tem uma importância expressiva na saúde pública, visto que tendem a persistir na fase adulta, podendo levar a incapacidades, impactos sociais e econômicos (Patel, Flisher, Hetrick \& McGorry, 2007). Por conseguinte, é fundamental o reconhecimento e a intervenção precoce como ferramentas para evitar a progressão dos sintomas.

Os alunos da Escola de Formação de Oficiais da Marinha Mercante (EFOMM), no Rio de Janeiro, estão suscetíveis a diversos fatores característicos da vida de um estudante em uma escola de formação militar que podem influenciar na saúde mental, tais como o distanciamento da família, a familiarização com a rotina, a hierarquia militar e o pouco tempo de descanso. Além disso, há os desafios comuns aos estudantes universitários, como maiores demandas acadêmicas, sociais, mudança ambiental e traços da transição do ensino médio para o nível superior (Gomes, Pereira Junior, Cardoso \& Silva, 2020). Portanto, investigar a saúde mental desse grupo específico é importante para o planejamento de medidas de prevenção e promoção à saúde. Em adição, durante o período do estudo, a pandemia da COVID-19 iniciava uma repercussão maior na cidade do Rio de Janeiro, com crescimento progressivo do número de casos e mortes, o que desencadeou novos estressores e, portanto, ameaças também à saúde mental das pessoas (Filgueiras \& Stults-Kolehmainen, 2020; Qiu et al., 2020).

Este estudo tem como objetivo investigar a presença de transtornos mentais comuns em alunos de todos os períodos da EFOMM, de acordo com suas características sociodemográficas e comportamentais.

\section{Metodologia}

Trata-se de um estudo transversal com uma abordagem quantitativa, realizado entre os meses de março e abril de 2020, com 194 alunos da Escola de Formação de Oficiais da Marinha Mercante (EFOMM), no Centro de Instrução Almirante Graça Aranha (CIAGA), localizado em Olaria, no Rio de Janeiro.

A abordagem quantitativa baseia-se em instrumentos de coleta de dados que implicam na codificação desses dados pelo processo de quantificação. Esse tipo de pesquisa busca a objetividade da análise estatística (Gaya, 2016).

Para avaliar a presença de TMC foi aplicada a versão brasileira do General Health Questionnaire versão de 12 itens (GHQ-12) (Goldberg, 1972), um instrumento autoadministrado utilizado para o rastreamento de transtornos não psicóticos. A versão adaptada para a população brasileira usada no presente estudo foi submetida a um estudo de validação com uma entrevista psiquiátrica como padrão-ouro. Como resultado, o questionário obteve $85 \%$ de sensibilidade e $79 \%$ de especificidade (Mari \& 
Williams, 1985). Cada item do GHQ-12 possui 4 opções de respostas referentes a um período de 15 dias anteriores ao preenchimento do questionário. Inicialmente, a pontuação individual foi classificada como "ausente" ou "presente" após a recodificação das opções de respostas em 001 1, respectivamente, e então somadas (Goldberg, 1972). Em consonância com outros estudos (Lopes et al.; Bastos, Barros, Celeste, Paradies \& Faerstein, 2014), o ponto de corte de 3 itens ou mais foi utilizado, de modo que os alunos que obtiveram pontuações iguais ou superiores ao ponto de corte estabelecido foram identificados como casos de TMC.

Os dados sociodemográficos foram coletados através de um questionário semiestruturado que contemplava as seguintes variáveis de interesse: sexo, idade, orientação sexual, cor da pele/raça autorreferida (após a coleta de dados, optou-se por retirar a cor da pele/raça indígena devido à ausência de alunos nessa categoria), ano escolar, posição socioeconômica e fé. As características socioeconômicas foram determinadas com base no modelo de questionário sugerido pela Associação Brasileira de Empresas de Pesquisa (ABEP) (2019), de acordo com o Critério de Classificação Econômica Brasil (CCEB). A idade foi coletada em anos e categorizada em faixas de 17 a 21 anos e 22 a 25 anos.

A variável orientação sexual foi coletada através de uma pergunta aberta e surgiram as seguintes respostas: pansexual, bissexual, heterossexual e homossexual. Para fins de análise de dados, a variável foi categorizada em heterossexual e não heterossexual. A fé foi avaliada a partir de uma pergunta sobre a intensidade (nada, moderadamente, muita) na qual exerce influência na vida do participante.

Todos os alunos participantes foram orientados quanto à pesquisa e apresentados a um termo de consentimento livre e esclarecido. $\mathrm{O}$ termo foi assinado e devolvido previamente à participação na pesquisa.

\section{Resultados e Discussão}

O Quadro 1 apresenta as características dos participantes do estudo e é composta por 194 alunos, com idade entre 17 e 25 anos. Do total, mais de 72\% dos alunos pertencem ao sexo masculino, sendo esse número quase 3 vezes maior que o de participantes do sexo feminino. Também são maioria na população de estudo os alunos entre 17 e 21 anos (61.9\%), os heterossexuais (90.7\%), os que autorreferem cor da pele branca (56.7\%), aqueles cursando o primeiro ano (39,7\%) e os de posição socioeconômica B2 (31.4\%). A frequência geral de transtornos mentais comuns entre os participantes foi de $71.1 \%$. 
Quadro 1. Características da amostra do estudo. (n=194)

\begin{tabular}{|c|c|}
\hline Variáveis & $\begin{array}{c}\text { Total } \\
\mathrm{n}=194(\%)\end{array}$ \\
\hline \multicolumn{2}{|l|}{ Sexo } \\
\hline Feminino & $54(27,8 \%)$ \\
\hline Masculino & $140(72,2 \%)$ \\
\hline \multicolumn{2}{|l|}{ Idade } \\
\hline Entre 17 e 21 anos & $120(61,9 \%)$ \\
\hline Entre 22 e 25 anos & $74(38,1 \%)$ \\
\hline \multicolumn{2}{|l|}{ Ano escolar } \\
\hline $1^{\circ}$ ano & $77(39,7 \%)$ \\
\hline $2^{\circ}$ ano & $64(33,0 \%)$ \\
\hline $3^{\circ}$ ano & $53(27,3 \%)$ \\
\hline \multicolumn{2}{|l|}{ Cor da pele } \\
\hline Branca & $110(56,7 \%)$ \\
\hline Parda & $62(32,0 \%)$ \\
\hline Preta & $20(10,3 \%)$ \\
\hline Amarela (Oriental) & $2(1,0 \%)$ \\
\hline \multicolumn{2}{|c|}{ Posição socioeconômica } \\
\hline A & $58(29,9 \%)$ \\
\hline B1 & $55(28,4 \%)$ \\
\hline $\mathrm{B} 2$ & $61(31,4 \%)$ \\
\hline $\mathrm{C} 1$ & $15(7,7 \%)$ \\
\hline $\mathrm{C} 2$ & $5(2,6 \%)$ \\
\hline \multicolumn{2}{|l|}{ Orientação sexual } \\
\hline Pansexual & $2(1,0 \%)$ \\
\hline Bissexual & $14(7,3 \%)$ \\
\hline Heterossexual & $176(90,7 \%)$ \\
\hline Homossexual & $2(1,0 \%)$ \\
\hline \multicolumn{2}{|c|}{ Influência da fé na vida } \\
\hline Nada & $28(14,4 \%)$ \\
\hline Moderadamente & $92(47,4 \%)$ \\
\hline Muito & $74(38,1 \%)$ \\
\hline \multicolumn{2}{|l|}{ Frequência de TMC* } \\
\hline Sim & $138(71,1 \%)$ \\
\hline Não & $56(28,9 \%)$ \\
\hline
\end{tabular}

*TMC - Transtorno Mental Comum. Fonte: Autores.

O Quadro 2 apresenta a frequência de TMC segundo sexo, idade, orientação sexual, cor da pele, ano escolar, posição socioeconômica e influência da fé na vida. Nota-se que, apesar da menor quantidade de mulheres no estudo (27.8\%), houve uma diferença estatisticamente significativa na proporção de TMC entre os sexos, sendo maior entre as jovens do sexo feminino $(85,2 \%)$ em comparação aos indivíduos do sexo masculino (65,7\%). Em relação a idade, nota-se que os jovens da faixa etária mais velha, entre 22 e 25 anos, apresentam maior frequência de TMC (83,4\%), também com significância estatística.

A frequência de TMC apresentou maiores índices entre os não heterossexuais (homossexuais, bissexuais e pansexuais), que compõem apenas $9.3 \%$ da população da amostra e, em relação a cor da pele, os TMCs se mostraram expressivos entre os participantes autodeclarados amarelos (100\%) e pretos (85\%), que correspondem a 11,3\% dos alunos. No entanto, em ambos os casos, essas diferenças não apresentaram significância estatística. 
Quadro 2. Frequência de TMC de acordo com as variáveis.

\begin{tabular}{|l|l|l|}
\hline Variáveis & $\begin{array}{l}\mathrm{n}=194 \\
(\%)\end{array}$ & $\mathrm{p}$-valor* \\
\hline Sexo & & \\
\hline Feminino & 85,2 & 0,007 \\
\hline Masculino & 65,7 & \\
\hline Idade & & \\
\hline Entre 17 e 21 anos & 64,2 & 0,006 \\
\hline Entre 22 e 25 anos & 83,4 & \\
\hline Ano escolar & & \\
\hline $1^{\mathbf{o}}$ ano & 68,8 & 0,830 \\
\hline $2^{\mathbf{o}}$ ano & 73,4 & \\
\hline $3^{\mathbf{o}}$ ano & 71,7 & \\
\hline Cor da pele & & \\
\hline Branco & 70,0 & 0,376 \\
\hline Pardo & 67,7 & \\
\hline Preto & 85,0 & \\
\hline Amarelo (Oriental) & 100,0 & \\
\hline Posição socioeconômica & & \\
\hline A & 63,8 & 0,622 \\
\hline B1 & 74,6 & \\
\hline B2 & 72,1 & \\
\hline C1 & 80,0 & \\
\hline C2 & 80,0 & \\
\hline Orientação sexual & & \\
\hline Heterossexual & 69,9 & 0,230 \\
\hline Não heterossexual & 83,3 & \\
\hline Influência da Fé na vida & & \\
\hline Nada & 67,9 & 0,870 \\
\hline Moderadamente & 70,7 & \\
\hline Muito & 73,0 & \\
\hline & & \\
\hline pocéa & & \\
\hline
\end{tabular}

*O p-valor é associado aos testes de Qui-quadrado entre o TMC e as variáveis estudadas. Fonte: Autores.

Na relação entre TMC e fé é observado um aumento gradativo da presença do transtorno conforme aumenta a influência da fé na vida do participante, sendo a maior frequência (73\%) entre aqueles que relataram maior intensidade. Outro gradiente de tendência parece ocorrer em relação ao estrato socioeconômico, onde a frequência de TMC aumenta à medida que a posição socioeconômica diminui.

Os achados deste estudo apontam para uma frequência de TMC expressiva entre os alunos, principalmente entre as alunas do sexo feminino. De forma geral, os TMC aparecem em todas as diferentes classes sociodemográficas e econômicas. No entanto, as maiores frequências estão presentes entre os grupos mais vulneráveis, como não brancos, não heterossexuais e menos favorecidos economicamente, o que traduz as possíveis dificuldades que estes podem sofrer ao longo da vida pessoal e profissional.

Devido à escassez de pesquisas epidemiológicas conduzidas em centros de instrução militar brasileiros, não foi possível comparar os resultados com outras instituições semelhantes. No entanto, a frequência de TMC encontrada entre os alunos (71.1\%) é maior do que a faixa de $35.7 \%$ a $50.9 \%$ encontrada em pesquisas conduzidas em instituições de ensino superior civis brasileiras (Bastos et al., 2014; Ansolin, Rocha, Santos \& Dal Pozzo, 2015; Silva \& Cavalcante, 2014). Tal fato pode não estar associado apenas à característica militar do centro de instrução, mas também ao período de quarentena implantado em decorrência da pandemia de COVID-19, que coincidiu com o período de aplicação dos questionários. Já é consolidado na literatura que, durante surtos de doenças, o medo de contrair tal enfermidade se torna um fator estressor (Filgueiras \& Stults, 
2020; Qiu et al., 2020; Brooks et al., 2020). O novo coronavírus, por ter sido descoberto recentemente, ainda não é completamente compreendido pela comunidade científica e por isso há um receio ainda maior no período atual.

A rotina de um centro de instrução militar envolve a permanência no local durante a semana e a autorização para sair aos finais de semana. A quarentena instituída devido à pandemia levou à suspensão da liberação para casa aos finais de semana. Portanto, os alunos permaneceram no centro de instrução durante 45 dias ininterruptos. Durante este período, o confinamento, a separação dos familiares, a perda da liberdade e o pouco contato com o meio exterior foram fatores chaves na deterioração da saúde mental dos indivíduos. Destaca-se, porém, que houve esforços para tornar o momento mais tolerável, através de sessões de filmes e apresentações musicais entre os alunos, o que auxiliaria afastar emoções prejudiciais, como o desânimo, ansiedade e a tristeza causados pelo distanciamento da família. Além disso, as instalações do CIAGA possibilitam a prática de inúmeros esportes e atividade físicas ao ar livre, os quais podem contribuir para amenizar os sintomas dos TMC tanto em jovens adultos e universitários (Silva \& Cavalcante, 2014; Calfas \& Taylor, 1994) quanto em militares (Oliveira et al., 2019).

O segundo e terceiro ano apresentaram frequências de TMC similares (73.4\% e 71.7\%, respectivamente) e mais altas em comparação com as frequências do primeiro ano (68.8\%). Reflete-se que, apesar da carga acadêmica ser maior entre os alunos que recém ingressaram na instituição, os que se encontram próximos da formatura se preocupam mais com a disponibilidade e economia do mercado mercante, o qual se encontra ainda mais instável devido à pandemia. Em consonância com a literatura, ainda podemos acrescentar a idade mais avançada dos alunos das turmas do terceiro e segundo ano como fator de risco para o desenvolvimento de transtornos depressivos e ansiosos. De acordo com Bonadiman, Passos, Mooney, Naghavi e Melo (2017), em um estudo descritivo da carga de transtornos mentais no Brasil, a carga de TMC aumenta conforme a idade e atinge seu pico na idade adulta em ambos os sexos. Vale destacar que a última turma a participar do estudo foi a do segundo ano, com uma diferença de aproximadamente uma semana e meia das outras duas, o que pode significar que, ao responder o questionário, a turma já estava há mais tempo exposta à influência da quarentena em sua saúde mental.

Um achado significativo no estudo foi a maior frequência de TMC entre o sexo feminino (85.2\%) quando comparado ao sexo masculino (65.7\%), o que é consistente com os dados do Estudo de Riscos Cardiovasculares em Adolescentes (ERICA), feito com 74.589 adolescentes de 1.247 escolas em 124 municípios brasileiros, em que a prevalência de TMC entre meninas foi de 38,4\% e de 21,6\% entre os meninos (Lopes et al., 2016). O estudo também tem resultados semelhantes ao estudo de Pinheiro et al. (2007), feito a partir da base populacional de adolescentes residentes em Pelotas, em que a prevalência de TMC entre meninas (37.2\%) era maior do que entre os meninos (19.9\%). Ambos estudos utilizaram o GHQ-12 para avaliar a presença de TMC. Além disso, pesquisa realizada por Beiter et al. (2015), revelou que uma quantidade maior de mulheres, em comparação com homens, reportou vida acadêmica e imagem corporal como significantes fatores de estresse, evidenciando a diferença entre os sexos no modo como experienciam fatores sociais e ambientais (Allen, Marmot, WHO \& Fundação Calouste Gulbenkian, 2014).

Em concordância com outros estudos (Gomes et al.,2020; Ueno, Vaghela \& Nix, 2018), o grupo não heterossexual, que envolve os participantes bissexuais, homossexuais e pansexuais, apresentou frequência maior (83.3\%) em relação aos heterossexuais (69.9\%). Minorias sexuais são vulneráveis a sofrer preconceitos e discriminação e por isso é esperado que apresentem maior prevalência de sintomas de transtornos de humor e ansiedade (Meyer, 2003).

Corroborando ao encontrado na literatura brasileira sobre a relação entre cor da pele e saúde mental (Bastos et al., 2014; Lucchese, Sousa, Bonfin, Vera \& Santana, 2014), os maiores índices estão entre as pessoas que autorreferem cor da pele amarela (100\%) e preta (85\%). Em outro estudo conduzido por Lopes et al. (2016), com população de 74.589 adolescentes, foi encontrada maior prevalência de TMC em pessoas de raça/cor asiática ou indígena, tendo sido utilizado o General Health Questionnaire com ponto de corte de cinco pontos que indica casos mais graves (Riberto, Correa, Oliveira \& Cade, 2020). A discriminação 
racial é um importante fator associado a elevadas frequências de TMC, sendo as pessoas negras mais vulneráveis a sofrer discriminação de classe e gênero quando comparadas às pessoas brancas (Macinko, Mullachery, Proietti \& Lima-Costa, 2012).

A relação entre posição socioeconômica e sofrimento psíquico também foi observada em outros estudos, que por sua vez atestaram que níveis socioeconômicos mais baixos estão associados à maior presença de transtornos mentais (Macinko et al., 2012; Mirza \& Jenkins, 2004). Tal associação pode acontecer devido às condições inadequadas de vida, discriminação de classe e o estresse psicossocial a que a população de estratos socioeconômicos mais baixos estão submetidas (Bastos et al., 2014; Macinko et al., 2012). De acordo com Gonçalves et al. (2014), em estudo realizado com 1,857 pessoas, gênero e baixa renda são as variáveis mais relevantes associadas com transtornos mentais comuns.

Ao contrário do esperado, no presente estudo podemos notar a tendência de aumento da frequência de TMC à medida que a influência da fé decai. Na literatura, existe uma tendência acerca da espiritualidade enquanto fator de proteção contra o desenvolvimento de transtornos psíquicos (VanderWeele, Balboni \& Koh, 2017; Murakami \& Campos, 2012)' porém, de acordo com Lawrence, Oquendo e Stanely (2016), a religião possui várias dimensões (afiliação, participação e doutrina) e por isso é difícil associar quais aspectos da fé estão ligados à saúde mental. Durante a quarentena, devido ao isolamento dentro da instituição, a interação entre os alunos e os encontros religiosos ficaram limitados ao ambiente do CIAGA, que não contempla todas as religiões e crenças, o que pode explicar em parte os resultados encontrados.

O presente estudo deve ser considerado à luz de seus pontos fortes e limitações. Entre os pontos positivos destaca-se o uso do GHQ-12 que, além de ser um instrumento amplamente utilizado em vários países, possibilitou maior comparabilidade para os resultados, foi traduzido e validado para a população brasileira com boas propriedades psicométricas (Mari \& Williams, 1985).

O período em que o estudo foi realizado pode ser considerado uma limitação, já que o período de pandemia foi relatado como estímulo ao sofrimento psíquico e por isso não é possível afirmar que em outro contexto os resultados seriam tão expressivos. Apesar disso, o estudo serve como contribuição para a compreensão de fatores de vulnerabilidade para o desenvolvimento de transtornos mentais comuns.

\section{Considerações Finais}

A taxa de TMC que predomina entre os estudantes na Escola de Formação de Oficiais da Marinha Mercante, com base na pesquisa efetuada, demonstra certa conformidade com estudos análogos desenvolvidos em outros ambientes. Apesar da interferência da COVID-19 provavelmente ter resultado em um aumento dos índices de sofrimento psíquico na população do estudo, os resultados obtidos sugerem que os sintomas depressivos e ansiosos já eram expressivos, o que pode implicar em problemas sérios não só na saúde mental como também na aprendizagem. Além disso, podem persistir ao longo da vida profissional dos alunos, com impactos negativos importantes. Portanto, a identificação precoce dos transtornos mentais comuns, bem como a divulgação do perfil epidemiológico diferencial entre os jovens, pode ser uma importante ferramenta de gestão para estratégias de ação em saúde mental que priorize grupos mais vulneráveis no ambiente universitário.

Sugere-se que haja mais pesquisas nessa área a fim de compreender melhor a saúde mental nesses grupos em Instituições Militares. O aumento de estudos com esse perfil pode contribuir para ampliar as ferramentas, estratégias de prevenção e intervenção quando for necessário. Esse é um campo que pode ser explorado de diversas formas promovendo o reconhecimento inicial da situação e conscientização dos estudantes, militares e profissionais de saúde sobre a importância do tema.

\section{Referências}

Agathão, B. T., Lopes, C. S., Cunha, D. B., \& Sichieri, R. (2020). Gender differences in the impact of sleep duration on common mental disorders in school students. BMC Public Health, 20(1), 148. https://doi.org/10.1186/s12889-020-8260-5 
Ansolin, A. G. A., Rocha, D. L. B., Santos, R. P. dos, \& Dal Pozzo, V. C. (2015). Prevalência de transtorno mental comum entre estudantes de psicologia e enfermagem. Revista Arquivos de Ciências da Saúde, 22(3), 42. https://doi.org/10.17696/2318-3691.22.3.2015.83

Associação brasileira de empresas de pesquisa. (2019). Critério Brasil: Critério de Classificação Econômica Brasil. São Paulo: ABEP.

Bastos, J. L., Barros, A. J. D., Celeste, R. K., Paradies, Y., \& Faerstein, E. (2014). Age, class and race discrimination: Their interactions and associations with mental health among Brazilian university students. Cadernos de Saúde Pública, 30(1), 175-186. https://doi.org/10.1590/0102-311X00163812

Beiter, R., Nash, R., McCrady, M., Rhoades, D., Linscomb, M., Clarahan, M., \& Sammut, S. (2015). The prevalence and correlates of depression, anxiety, and stress in a sample of college students. Journal of Affective Disorders, 173, 90-96. https://doi.org/10.1016/j.jad.2014.10.054

Bonadiman, C. S. C., Passos, V. M. de A., Mooney, M., Naghavi, M., \& Melo, A. P. S. (2017). A carga dos transtornos mentais e decorrentes do uso de substâncias psicoativas no Brasil: Estudo de Carga Global de Doença, 1990 e 2015. Revista Brasileira de Epidemiologia, 20(suppl 1), 191-204. https://doi.org/10.1590/1980-5497201700050016

Brooks, S. K., Webster, R. K., Smith, L. E., Woodland, L., Wessely, S., Greenberg, N., \& Rubin, G. J. (2020). The psychological impact of quarantine and how to reduce it: Rapid review of the evidence. The Lancet, 395(10227), 912-920. https://doi.org/10.1016/S0140-6736(20)30460-8

Calfas, K. J., \& Taylor, W. C. (1994). Effects of physical activity on psychological variables in adolescents. Pediatric Exercise Science, 6(4), 406-423. https://doi.org/10.1123/pes.6.4.406

De Jesus Mari, J., \& Williams, P. (1985). A comparison of the validity of two psychiatric screening questionnaires (GHQ-12 and SRQ-20) in Brazil, using Relative Operating Characteristic (Roc) analysis. Psychological Medicine, 15(3), 651-659. https://doi.org/10.1017/S0033291700031500

Filgueiras, A., \& Stults-Kolehmainen, M. (2020). The relationship between behavioural and psychosocial factors among brazilians in quarantine due to covid19 (SSRN Scholarly Paper No ID 3566245). Recuperado de Social Science Research Network website: https://papers.ssrn.com/abstract=3566245

Gaya, Adroaldo. Projetos de pesquisa científica e pedagógica: o desafio da iniciação científica. Casa da Educação Física, 2016. 426p.

Goldberg, D. (1994). A bio-social model for common mental disorders. Acta Psychiatrica Scandinavica, 90(s385), 66-70. https://doi.org/10.1111/j.16000447.1994.tb05916.x

Goldberg, D. P. (1972). The detection of psychiatric illness by questionnaire: A technique for the identification and assessment of non-psychotic psychiatric illness. London: Oxford University Press.

Gomes, C. F. M., Pereira Junior, R. J., Cardoso, J. V., \& Silva, D. A. da. (2020). Transtornos mentais comuns em estudantes universitários. SMAD Revista Eletrônica Saúde Mental Álcool e Drogas (Edição em Português), 16(1), 1-8. https://doi.org/10.11606//issn.1806-6976.smad.2020.157317

Gonçalves, D. A., Mari, J. de J., Bower, P., Gask, L., Dowrick, C., Tófoli, L. F., ... Fortes, S. (2014a). Brazilian multicentre study of common mental disorders in primary care: Rates and related social and demographic factors. Cadernos de Saúde Pública, 30(3), 623-632. https://doi.org/10.1590/0102-311X00158412

Grether, E. O., Becker, M. C., Menezes, H. M., \& Nunes, C. R. de O. (2019). Prevalência de transtornos mentais comuns entre estudantes de medicina da Universidade Regional de Blumenau(SC). Revista Brasileira de Educação Médica, 43(1 suppl 1), 276-285. https://doi.org/10.1590/1981-5271v43suplemento120180260

Ibrahim, A. K., Kelly, S. J., Adams, C. E., \& Glazebrook, C. (2013). A systematic review of studies of depression prevalence in university students. Journal of Psychiatric Research, 47(3), 391-400. https://doi.org/10.1016/j.jpsychires.2012.11.015

Lawrence, R. E., Oquendo, M. A., \& Stanley, B. (2016). Religion and suicide risk: A systematic review. Archives of Suicide Research, 20(1), 1-21. https://doi.org/10.1080/13811118.2015.1004494

Lopes, C. S., Abreu, G. de A., Santos, D. F. dos, Menezes, P. R., Carvalho, K. M. B. de, Cunha, C. de F., \& Szklo, M. (2016). ERICA: Prevalence of common mental disorders in Brazilian adolescents. Revista de Saúde Pública, 50(suppl 1). https://doi.org/10.1590/s01518-8787.2016050006690

Lucchese, R., Sousa, K. de, Bonfin, S. do P., Vera, I., Santana, F. R., Lucchese, R., ... Santana, F. R. (2014). Prevalência de transtorno mental comum na atenção primária. Acta Paulista de Enfermagem, 27(3), 200-207. https://doi.org/10.1590/1982-0194201400035

Macinko, J., Mullachery, P., Proietti, F. A., \& Lima-Costa, M. F. (2012). Who experiences discrimination in Brazil? Evidence from a large metropolitan region. International Journal for Equity in Health, 11(1), 80. https://doi.org/10.1186/1475-9276-11-80

Marinho, F., de Azeredo Passos, V. M., Carvalho Malta, D., Barboza França, E., Abreu, D.M. X., Araújo, V. E. M., \& Naghavi, M. (2018). Burden of disease in Brazil, 1990-2016: A systematic subnational analysis for the Global Burden of Disease Study 2016. The Lancet, 392(10149), 760-775. https://doi.org/10.1016/S0140-6736(18)31221-2

Meyer, I. H. (2003). Prejudice, social stress, and mental health in lesbian, gay, and bisexual populations: Conceptual issues and research evidence. Psychological Bulletin, 129(5), 674-697. https://doi.org/10.1037/0033-2909.129.5.674

Mirza, I., \& Jenkins, R. (2004). Risk factors, prevalence, and treatment of anxiety and depressive disorders in Pakistan: Systematic review. BMJ, 328(7443), 794. https://doi.org/10.1136/bmj.328.7443.794

Murakami, R., \& Campos, C. J. G. (2012). Religião e saúde mental: Desafio de integrar a religiosidade ao cuidado com o paciente. Revista Brasileira de Enfermagem, 65(2), 361-367. https://doi.org/10.1590/S0034-71672012000200024

Oliveira, A. J., Neto, G. A. M., Barros, O. D., Pedreiro, R., Murillo-Rodriguez, E., de Leon, A. C. P., \& Machado, S. (2019). Association between physical fitness and psychologicaldistress among Brazilian armed force personnel. Sport Sciences for Health, 15(1), 141-147. https://doi.org/10.1007/s11332-018-0503- 
Patel, V., Flisher, A. J., Hetrick, S., \& McGorry, P. (2007). Mental health of young people: A global public-health challenge. The Lancet, 369(9569), 13021313. https://doi.org/10.1016/S0140-6736(07)60368-7

Pinheiro, K. A. T., Horta, B. L., Pinheiro, R. T., Horta, L. L., Terres, N. G., \& Silva, R. A. da. (2007). Common mental disorders in adolescents: A population based cross-sectional study. Revista Brasileira de Psiquiatria, 29(3), 241-245. https://doi.org/10.1590/S1516-44462006005000040

Qiu, J., Shen, B., Zhao, M., Wang, Z., Xie, B., \& Xu, Y. (2020). A nationwide survey of psychological distress among Chinese people in the COVID-19 epidemic: Implications and policy recommendations. General Psychiatry, 33(2), e100213. https://doi.org/10.1136/gpsych-2020-100213

Ribeiro, I. B. da S., Correa, M. M., Oliveira, G., \& Cade, N. V. (2020). Common mental disorders and socioeconomic status in adolescents of ERICA. Revista de Saúde Pública, 54, 4. https://doi.org/10.11606/S1518-8787.2020054001197

Silva, A. G., Cerqueira, A. T. de A. R., \& Lima, M. C. P. (2014). Social support and common mental disorder among medical students. Revista Brasileira de Epidemiologia, 17(1), 229-242. https://doi.org/10.1590/1415-790X201400010018ENG

Silva, Adenice de Omena, \& Cavalcante Neto, Jorge Lopes. (2014). Associação entre níveis de atividade física e transtorno mental comum em estudantes universitários. Motricidade, 10(1), 49-59. https://dx.doi.org/10.6063/motricidade.10(1).2125

Steel, Z., Marnane, C., Iranpour, C., Chey, T., Jackson, J. W., Patel, V., \& Silove, D. (2014). The global prevalence of common mental disorders: A systematic review and meta-analysis1980-2013. International Journal of Epidemiology, 43(2), 476-493. https://doi.org/10.1093/ije/dyu038

Ueno, K., Vaghela, P., \& Nix, A. N. (2018). Gender composition of the occupation, sexual orientation, and mental health in young adulthood. Stress and Health, 34(1), 3-14. https://doi.org/10.1002/smi.2755

VanderWeele, T. J., Balboni, T. A., \& Koh, H. K. (2017). Health and spirituality. JAMA, 318(6), 519. https://doi.org/10.1001/jama.2017.8136

World Health Organization \& Calouste Gulbenkian Foundation. (2014). Social Determinants of Mental Health. World Health Organization.

World Health Organization. (2017). Depression and Other Common Mental Disorders: Global Health Estimates. World Health Organization 